\title{
Solos urbanos
}

\author{
Urban soils \\ Fabrício de Araújo Pedron ${ }^{1}$ Ricardo Simão Diniz Dalmolin² \\ Antônio Carlos de Azevedo ${ }^{3}$ João Kaminski ${ }^{4}$
}

\section{- REVISÃO BIBLIOGRÁFICA -}

\section{RESUMO}

A forte pressão provocada pela expansão urbana desordenada sobre os recursos naturais, principalmente os solos, tem provocado danos, muitas vezes de difícil reparo. A grande concentração populacional em centros urbanos cada vez maiores tem dirigido a atenção de diferentes profissionais para o recurso solo, no sentido de entender sua dinâmica para minimizar sua degradação. No entanto, a falta de conhecimento sobre as propriedades, bem como sobre a aptidão dos solos sob uso urbano tem provocado o seu mau uso, resultando em processos como compactação, erosão, deslizamentos e inundações, assim como poluição com substâncias orgânicas, inorgânicas e patógenos, aumentando os custos do desenvolvimento afetando toda a sociedade. Neste sentido, este texto discute como o conhecimento pedológico pode diminuir os efeitos negativos provocados pelo processo de urbanização.

Palavras-chave: pedologia, levantamento de solos, classificação de solos, poluição dos solos, planejamento de uso dos solos urbanos, paisagismo.

\section{ABSTRACT}

The strong pressure caused by the disordered urban expansion over the natural resources, mainly the soils, has caused damages, many times difficult to repair. The great population concentration in urban centers getting larger and larger has been driving the attention of different professionals to soil resource, in the sense of understanding its dynamics to minimize its degradation. The lack of knowledge related to the soils properties and capability promote their inappropriate use, resultig in degrading processes as compaction, erosion, sliding, floods, and organic, inorganic and patogenic pollution, increasing the cost of development and affecting the whole society. This text discusses how pedologic knowledge can reduce the negative effects caused by the urbanization process.
Key words: pedology, soil survey, soil classification, soil pollution, urban soils use planning, landscaping.

\section{INTRODUÇÃO}

Os solos são corpos naturais que se desenvolvem em escalas de tempo da ordem de centenas a milhares de anos, e compõem a cobertura pedológica que reveste as áreas emersas da Terra. Esta cobertura é constituída por uma camada de material alterado que se localiza entre a atmosfera e a litosfera, fortemente influenciada pela biosfera e pela hidrosfera. Esta camada é o resultado das inúmeras combinações de fatores (clima, organismos, tempo, relevo) e de processos (remoção, adição, transporte e transformação) que atuam sobre os materiais de origem (rochas, sedimentos, depósitos orgânicos) e condicionam a variedade de solos encontrados. Inferências sobre as propriedades destes corpos são feitas, entre outras características, a partir de sua morfologia, por exemplo, através da presença e espessura de horizontes ou camadas, representando o perfil do solo.

O solo não é uma entidade discreta, isto é, não há um indivíduo solo na natureza, mas um contínuo na paisagem, com progressiva gradação resultante da combinação dos processos e fatores pedogenéticos (JENNY, 1941). Os processos de transformações químicas, físicas e biológicas que ocorrem no solo são

\footnotetext{
'Engenheiro Agrônomo, Aluno do curso de mestrado do Programa de Pós-graduação em Ciência do Solo, Universidade Federal de Santa Maria, UFSM, Autor correspondente, Rua Ilaquídio Rasquin, 25 apt. 303 D, 97060-660, Santa Maria, RS. E-mail: fapedron@yahoo.com.

${ }^{2}$ Engenheiro Agrônomo, Doutor Professor Adjunto do Departamento de Solos, Centro de Ciências Rurais (CCR), UFSM.

${ }^{3}$ Engenheiro Agrônomo, PhD, Professor Adjunto do Departamento de Solos, CCR, UFSM.

${ }^{4}$ Engenheiro Agrônomo, Doutor, Professor, Departamento de Solos, CCR, UFSM.
} 
de interesse dos profissionais das mais diversas áreas da ciência, como por exemplo, agronomia, geologia, geografia, engenharia civil e engenharia ambiental. $\mathrm{O}$ profissional especialista na caracterização, identificação, classificação e mapeamento dos solos é o pedólogo, que atua com outros profissionais na solução de problemas e no planejamento do uso deste recurso natural. Historicamente, os grupos de pedólogos se concentram em sua maioria em instituições de pesquisa e ensino agrícola , centralizando a pesquisa pedológica em questões desta área. À medida em que os problemas relacionados ao mau uso urbano do solo se avolumam, e são alocados recursos para estudos, uma nova frente de pesquisa e desenvolvimento de tecnologias emerge. A identificação, comportamento e uso dos solos em meio urbano possuem um caráter complexo, requerendo a participação de profissionais das mais diversas áreas, tanto na produção quanto no uso das informações.

A artificialidade da divisão da ciência em áreas distintas se materializa, entre outras maneiras, na diversidade de conceitos e vocabulário para objetos e processos semelhantes. Neste contexto, o termo "solos urbanos" tem sido empregado com freqüência crescente, porém de maneira diversa e com significados variados. Portanto, o objetivo deste texto é discutir: 1. o uso do termo "solos urbanos"; 2. algumas das funções que os solos desempenham no meio urbano; e 3. as modificações mais freqüentes impostas ao solo pela dinâmica das cidades, visando introduzir o tema dos solos sob uso urbano, principalmente para profissionais sem formação específica em Ciência do Solo.

\section{CONCEITUAÇÃO DOS SOLOS URBANOS}

O termo "solos urbanos" refere-se a solos que se encontram no meio urbano e tem sido referenciado frequientemente em artigos científicos de revistas internacionais (CRAUL, 1992; STROGANOVA \&AGARKOVA, 1993; JIM, 1998; CRAUL, 1999; USDA, 2000a; GE et al., 2000; DE KIMPE \& MOREL, 2000; MADRID et al., 2002; MANTA et al., 2002; LU et al., 2003;). A Sociedade Internacional de Ciência do Solo (ISSC) também tem empregado este termo com frequiência com especial atenção nos últimos dois congressos mundiais de ciência do solo (França, 1998 e Tailândia, 2002) em que houve simpósios específicos para discutir a natureza, manejo e riscos a saúde humana dos solos urbanos (urban soils). Desta forma, o termo "solos urbanos" teria a função de ressaltar o uso do solo e apontar para um conjunto de possíveis modificações nas suas propriedades, típicas do meio urbano, algumas discutidas adiante neste texto.
Mais da metade da população mundial vive em cidades, fato jamais ocorrido na historia da humanidade. Portanto, nada mais necessário que o solo seja estudado sob este tipo de uso, porém a maior dificuldade é distinguir entre as características pedogenéticas daquelas resultantes do uso urbano (BLUME, 1989).

É preciso destacar que os termos "solos antrópicos" e "solos urbanos" não são equivalentes. Solos antrópicos é um termo que contempla aqueles significantemente modificados pelo uso intenso e continuado do homem através da exploração agrícola, mineral, urbana, etc. A quase totalidade dos sistemas de classificação de solos no mundo são morfogenéticos (usam critérios morfológicos relacionados aos processos de formação dos solos para definir as classes de solos). Muitos deles são contemplados com o termo solos antrópicos, como no sistema da FAO, francês (SPAARGAREN, 2000), WRB (ISSSWG RB, 1998) e o australiano (ISBELL, 1996). O Sistema Brasileiro de Classificação de Solos (SiBCS) atualmente não contempla o termo solo antrópico, apenas horizonte A antrópico (EMBRAPA, 1999). Existe a proposição de se criar a ordem Antropossolos, a qual foi apresentada no XXIX Congresso Brasileiro de Ciência do Solo (2003).

Solos urbanos têm sido discutidos mundialmente como uma subdivisão dos solos antrópicos, como já ocorrem em alguns sistemas de classificação: sistema Russo (POPKOV \& DEMENT'EVA, 2002) e o sistema da FAO (FAO, 1994). Há uma grande dificuldade na definição de critérios para classificação dos solos urbanos, já que a atividade humana é bastante complexa, podendo esta ser detectada pela alta concentração de metais pesados (ALEXANDROVSKAYA\&ALEXANDROVSKIY, 2000; HILLER, 2000; MADRID et al., 2002; MANTAet al., 2002; NAVAS \& MACHIN, 2002), metano (BLUME, 1989), deposição de rejeitos de construção e industriais (STROGANOVA \& AGARKOVA, 1993; ALEXANDROVSKAYA\&ALEXANDROVSKIY,2000), e/ou alteração do regime hídrico e térmico do solo (STROGANOVA\&AGARKOVA, 1993). Esta variedade de efeitos dificulta o estabelecimento de uma metodologia para levantamentos de solos em meio urbano.

Portanto, não resta dúvida da necessidade de incluir solos urbanos nos sistemas de classificações taxonômicas. Uma vez que estes sistemas são a base para os sistemas interpretativos e para elaboração dos mapas de solos, tal inclusão representa um salto na capacidade de definir a vocação de uso (urbana) dos solos, contribuindo no planejamento das cidades. 


\section{FUNÇÕES DOSOLONO MEIOURBANO}

As principais funções desempenhadas pelos solos no meio urbano são: suporte e fonte de material para obras civis, sustento das agriculturas urbanas, suburbanas e de áreas verdes, meio para descarte de resíduos e armazenamento e filtragem de águas pluviais.

Para que o solo desempenhe cada uma destas funções, determinadas propriedades pedológicas devem ser funcionais e em ressonância com outras propriedades externas ao solo. Por exemplo, a propriedade do solo "porosidade" determina a taxa e o volume total de água que o solo pode reter durante uma chuva, retardando e diminuindo o pico de vazão dos cursos de água. O processo de urbanização pode compactar o solo, diminuindo a porosidade e a infiltração de água, aumentando o escorrimento superficial, de modo que um volume maior de precipitação escorre mais rapidamente para os cursos de água, aumentando o pico de vazão e o potencial de enchentes. O processo de urbanização sem planejamento em relação ao recurso solo acentua as alterações morfológicas, a compactação,a erosão, a poluição por substâncias tóxicas, vetorização de doenças e o deslizamento de encostas. Desnecessário é discorrer sobre as perdas materiais e humanas, a redução na qualidade de vida e o custo de recuperação destes eventos.

Em conseqüência, cada vez mais se torna consenso que os levantamentos de solos devem abranger as áreas urbanas (D'COSTA et al., 2002). No processo de urbanização planejado, a aptidão de uso dos solos deve ser considerada, observando suas potencialidades e respeitando suas limitações e fragilidades. O conhecimento do solo é fundamental, por exemplo, para determinar as condições para o desenvolvimento de plantas, situação do lençol freático, inertização de substâncias tóxicas (SCHLEUß et al., 1998) e capacidade de suporte de obras civis (OLIVEIRA, 2002). Os levantamentos de solos nas áreas urbanas e em seu entorno devem então ser vistos como uma importante ferramenta na tomada de decisões para a expansão urbana ou recuperação de espaços urbanos degradados (KELLER, 1996).

Suporte para obras civis

Para especialidades como a engenharia civil o solo é um material sobre o qual são edificados os mais diversos tipos de obras, devendo este apresentar propriedades que permitam sua sustentação. Diversos parâmetros geotécnicos devem ser observados como adensamento, permeabilidade, resistência ao cisalhamento, erodibilidade, colapsividade, resistência compactada e saturada, compressibilidade compactada e saturada, entre outras (OLIVEIRA \& BRITO, 1998). A questão negativa da expansão urbana é relativa a artificialização do ambiente. Há, porém, uma prática crescente entre arquitetos e engenheiros em se considerar a organização original do ambiente nos projetos de obras urbanas (por exemplo, OLIVEIRA, 2002), esbarrando na falta de informação sobre a aptidão de uso do solo no meio urbano e dos demais elementos que compõem o ambiente.

Agricultura urbana

O desenvolvimento de atividades agrícolas no meio urbano é bastante freqüente, sendo favorecida pela proximidade do mercado consumidor e disponibilidade de mão-de-obra (GRANDO \& MIGUEL, 2002). A produção de alimentos no meio urbano é de pequena escala, destacando-se a produção de frutas e culturas de ciclo rápido, como as hortaliças. Raramente as áreas usadas para este fim não sofreram alterações. Por isso, esta atividade pode oferecer riscos aos consumidores, quando implantada sobre solos já utilizados para descartes de resíduos e/ ou efluentes domésticos, o que pode promover sua contaminação. Apesar de risco semelhante estar potencialmente presente nas áreas agrícolas, nas áreas urbanas a concentração de produtos tóxicos tende a ser maior, e a destinação da produção para o consumo humano dá-se, em grande parte, diretamente. Outro fato importante em relação à agricultura urbana se relaciona ao tipo de manejo aplicado no sistema de produção, que pode oferecer riscos à população através de adubações incorretas do solo e aplicações inadequadas de produtos fitossanitários altamente tóxicos, aplicados sem acompanhamento técnico.

\section{Áreas verdes}

É nas áreas adjacentes às construções que se concentram os maiores problemas relacionados ao solo destinado às áreas verdes, acarretando limitações ao desenvolvimento da arborização urbana (HARRIS et al.,1999). De modo geral, as condições de solo favoráveis às atividades de engenharia se contrapõem àquelas adequadas ao crescimento das plantas. Em decorrência, a maioria das árvores encontradas nos centros urbanos apresenta tempo de vida muito menor que o potencial biológico de sua espécie, reduzindo a qualidade ambiental aumentando os custos da arborização (JIM, 1998). Tal fato é conseqüência, principalmente, da intensa modificação ocorrida na maioria dos solos do meio urbano, tornando-os inadequados a este fim (BRADSHAW et al., 1995; JIM, 
1998). Em vista disso os solos de áreas destinadas à arborização e ajardinamento devem permanecer o mais natural possível, evitando cortes, aterros, ou compactação excessiva, recebendo o mesmo tratamento agronômico prescrito para áreas não urbanas.

Descarte de resíduos

É muito freqüente encontrarmos, principalmente nos grandes centros urbanos, terrenos utilizados para o descarte de materiais oriundos de atividades antrópicas. A natureza do resíduo descartado é extremamente importante, determinando sua periculosidade em relação ao ambiente e às transformações promovidas no solo. É fundamental que o solo apresente propriedades físicas e químicas que o capacitem a inertizar os resíduos pelo maior tempo possível, evitando liberá-los ao ambiente, principalmente os materiais ricos em metais pesados, que deveriam ser descartados, em pequenas doses, sobre solos com alto teor de argila e matéria orgânica e alto $\mathrm{pH}$, com monitoramento técnico (GAIVIZZO, 2001).

Infiltração de águas pluviais

O crescimento urbano acelerado tem provocado um excesso de superfícies impermeabilizadas, que reduzem a infiltração de águas das chuvas, aumentando os riscos de erosão, compactação e deslizamentos de solos, bem como alagamentos de córregos e ruas (ROBAINA et al., 2001). A falta de planejamento urbano e cumprimento da legislação vigente favorecem a excessiva impermeabilização da superfície urbana pelo revestimento de terrenos e/ou pela compactação dos solos. Um sistema hídrico de captação pluvial eficiente deve considerar informações sobre os diferentes tipos de solos que ocorrem numa microbacia urbana, para que a drenagem adequada seja efetuada sem danos ambientais.

\section{MODIFICAÇÕES NO SOLO PELO USO URBANO ESUAS IMPLICAÇÕES}

As mudanças ocorridas no solo provocadas pela urbanização ainda não assumiram, em sua maioria, caráter pedogenético pela rapidez com que ocorrem em relação à escala temporal destes processos. É interessante que o conhecimento e experiência acumulados no uso agrícola e florestal do solo possa ser aproveitado ao máximo para compreender seu comportamento quando sob uso urbano. Assim, pelo menos algumas das modificações pelo uso urbano, como cortes e aterros e drenagem artificial são, em essência, semelhantes àquelas realizadas em algumas situações no meio rural (BLUME, 1989).
Alterações morfológicas nos solos urbanos

A influência antrópica nos solos encontrados no meio urbano pode provocar diversas alterações morfológicas (SCHLEUß et al., 1998). Em muitos casos, o horizonte superficial não é encontrado, tendo este já sido removido em áreas de corte, ou no caso de áreas de aterro, pode ocorrer sobreposição de camadas superficiais. É muito freqüente a ocorrência de camadas distintas e artificiais resultante da introdução de diferentes materiais, com diferentes texturas, devido à tentativa de reconstituição do solo removido, ou descarte de restos de construções sobre o terreno (JIM, 1998). As camadas também não apresentam transição plana ou ondulada, mas sim, transição irregular ou descontínua, justamente devido à adição de materiais exógenos, que nem sempre é homogênea em toda a área (DE KIMPE et al., 2000). Esta heterogeneidade morfológica do solo é importante, pois interfere no regime hídrico e térmico do solo, na sua capacidade de sustentação de plantas e na sua resistência à erosão e deslizamentos.

Compactação dos solos urbanos

$\mathrm{O}$ processo de compactação refere-se à compressão do solo não saturado promovendo o aumento da sua densidade e redução do seu volume, resultante da expulsão do ar dos poros do solo (DIAS JUNIOR, 2000). A compactação do solo pode ser acentuada se o mesmo for comprimido com teor de umidade alta (BAVER et al., 1972; REINERT, 1990). A compactação possui efeitos diretos sobre propriedades físicas do solo. A resistência mecânica do solo aumenta com a compactação (SOANE \& VAN OUWERKERK, 1994; DIAS JUNIOR, 2000), enquanto a porosidade total decresce, principalmente a macroporosidade (HILLEL, 1998; DIAS JUNIOR, 2000). Esta situação é desejada em obras de engenharia como estradas e barragens, porém, é muito prejudicial em locais a serem arborizados como parques, praças e quintais domésticos. De acordo com SCHUELER (2000), solos não compactados, em sua condição natural, possuem valores de densidade volumétrica que variam em média de $1,1 \mathrm{a} 1,4 \mathrm{~g} \mathrm{~cm}^{-3}$, enquanto solos urbanos geralmente apresentam valores entre $1,5 \mathrm{a} 1,9 \mathrm{~g}$ $\mathrm{cm}^{-3}$, podendo chegar até $2,0 \mathrm{~g} \mathrm{~cm}^{-3}$. A compactação dos solos em áreas urbanas ocorre em dois estágios: primeiro, quando toda a área é compactada com a finalidade de facilitar o tráfego de equipamentos, descarga de materiais, pavimentação do terreno e as condições para construção das fundações do prédio; segundo, quando a obra encontra-se finalizada, pelo tráfego sem controle de veículos e pedestres sobre as áreas verdes (USDA, 2000a). A compactação dificulta 
a implantação e manutenção de jardins devido à restrição ao desenvolvimento radicular e redução na absorção de água e nutrientes pelas plantas, e aumenta o escorrimento superficial devido à menor taxa de infiltração de água no solo, aumentando o processo erosivo (SCHUELER, 2000).

Erosão dos solos urbanos

A erosão é um fenômeno resultante da desagregação, transporte e deposição ou sedimentação das partículas de solos pela ação da chuva ou do vento. A erosão, geralmente, ocorre na superfície do solo removendo a porção mais fértil do perfil, onde há melhores condições biológicas e físicas ao desenvolvimento radicular das plantas (HUDSON, 1995; AGASSI, 1996; HILLEL, 1998). A erosão ocorre quando o solo permanece desnudo e exposto à ação abrasiva dos ventos e da água (CRAUL, 1999). Obras como cortes e aterros podem tornar estes locais mais suscetíveis à erosão. A retirada da mata ciliar, depósitos irregulares de lixo e alta impermeabilização da bacia urbana (ROBAINA et al., 2001) aumentam o transporte e sedimentação das partículas de solos (KELLER, 1996; USDA, 2000b) afetando a freqüência e intensidade das inundações em cursos d'água.

O controle da erosão em solos urbanos pode ser realizado através do agendamento dos trabalhos em épocas não chuvosas, redução do tempo de início e fim das obras de corte e aterro, cobertura do solo e controle do fluxo de água dentro do terreno (CRAUL, 1999). É inevitável que o solo fique exposto durante os trabalhos de construção, entretanto, é essencial que a área exposta seja minimizada, bem como o seu tempo de exposição (USDA, 2000b).

Assim como a erosão, os deslizamentos de solos são fenômenos naturais que atuam na formação da paisagem em relativo equilíbrio com o ecossistema. Entretanto, as atividades humanas não planejadas como a ocupação inadequada de solos (isto é, sem observância de sua aptidão de uso) têm acelerado intensamente estes fenômenos (BERGER, 2001), modificando o comportamento hidrológico do solo, favorecendo a instabilidade e seu deslizamento morro abaixo (KELLER, 1996). De acordo com Bernstein apud SMYTH \& ROYLE (2000) e ROBAINA et al. (2001), a falta de uma legislação apropriada e ações governamentais são responsáveis, em grande parte, pela freqüência destes acontecimentos.

A estabilidade de encostas depende da presença da vegetação protegendo o solo da ação das gotas de chuvas, favorecendo a infiltração de água e promovendo coesão entre as partículas de solo, aumentando sua resistência à erosão. Além disto, o uso de vegetação é mais eficiente e de menor custo.

\section{Poluição dos solos urbanos}

Segundo BRASIL (1981), o termo poluição é definido como toda alteração das propriedades físicas, químicas e biológicas que possa constituir prejuízo à saúde, à segurança e ao bem estar das populações e, ainda, possa comprometer a biota e a utilização dos recursos para fins comerciais, industriais e recreativos. Desta forma, a poluição do solo significa a presença de níveis de algum elemento ou substância que pode afetar componentes bióticos do ecossistema, comprometendo sua funcionalidade e sustentabilidade. Diferentemente dos solos agrícolas, os solos urbanos, particularmente em parques públicos e jardins residenciais, possuem influência na saúde humana pelo contato direto e freqüente com homem (MADRID et al., 2002; MANTA et al., 2002). Solos utilizados previamente em parques industriais podem conter grandes quantidades de poluentes minerais e orgânicos (DE KIMPE \& MOREL, 2000; MADRID et al., 2002). A poluição dos solos por metais pesados e substâncias tóxicas orgânicas e inorgânicas tem sido relatadas em muitos contextos diferentes, sendo essencial sua detecção para evitar problemas relacionados à saúde, bem como degradações ambientais (BERNARD, 1997; ACCIOLY \& SIQUEIRA, 2000).

Os poluentes orgânicos são de difícil identificação e seu efeito na saúde humana é pouco conhecido. Entre estas substâncias, destacam-se as derivadas de petróleo, encontradas em níveis geralmente altos no meio urbano (ACCIOLY \& SIQUEIRA, 2000).

Os poluentes inorgânicos mais freqüentes nos solos urbanos são os metais pesados, sendo os mais comuns o cobre, o chumbo, o zinco (MADRID et al., 2002), o cádmio e o níquel (GE et al., 2000). Estes elementos podem entrar na cadeia trófica, afetando plantas, animais, homens e poluindo os mananciais hídricos (ACCIOLY \& SIQUEIRA, 2000). Nas plantas, o excesso de metais pode provocar mudanças fisiológicas, redução do vigor ou até mesmo inibição do desenvolvimento vegetal (SIMÃO \& SIQUEIRA, 2001) dependendo da sua concentração e especiação química (GE et al., 2000; LU et al., 2003). A presença de altos teores de chumbo e mercúrio pode provocar sérios problemas no sistema nervoso central de fetos e crianças, assim como o cádmio pode provocar problemas renais (BERNARD, 1997).

Os metais pesados podem ser introduzidos no solo pela deposição atmosférica como o chumbo eliminado pela combustão dos veículos (JIM, 1998; SHINN et al., 2000; LU et al., 2003), pela utilização de 
lodos de estação de tratamento de esgoto urbano e industrial e também, pela deposição de rejeitos industriais, extração e processamento de minérios (ACCIOLY \& SIQUEIRA, 2000). Outra rota de poluição bastante comum é através da utilização de compostos orgânicos resultantes da reciclagem de lixo urbano, os quais podem conter grande quantidade de metais pesados (ANJOS et al., 2002).

Os solos como vetor de doenças

É grande o número de organismos que utilizam o solo como vetor na transmissão de doenças. Entre as principais podem ser citadas: ancilostomíase, ascaridíase, amebíase, cólera, diarréia infecciosa, disenteria bacilar, esquistossomose, estrongiloidíase, febre tifóide, febre paratifóide, salmonelose, teníase e cisticercose (FUNASA, 1992). O solo serve como suporte na manutenção do ciclo de vida de vários agentes causadores e transmissores de doenças como insetos, bactérias, protozoários, platelmintos, fungos e outros organismos. O contágio humano pode ocorrer pelo contato direto ou indireto com o patógeno encontrado em solos contaminados. A presença de dejetos de animais, esgotos, lixos e resíduos industriais orgânicos e inorgânicos no solo promove a contaminação direta, enquanto o contato indireto com o patógeno ocorre através do consumo de alimentos mal higienizados produzidos em solos contaminados.

\section{CONCLUSÕES}

Problemas como a compactação, a erosão, a poluição, inundações e deslizamentos no meio urbano podem ser provocados pela utilização inadequada do recurso solo, resultante da falta de conhecimento do seu comportamento quando submetido às aplicações urbanas. Portanto, o solo deve ser utilizado conforme sua aptidão de uso, observando suas potencialidades e respeitando suas limitações e fragilidades. Em todos estes eventos, há uma degradação da qualidade de vida, e em muitos, os prejuízos são irreparáveis ou sua recuperação é inviável. Neste sentido, é necessário maior investimento no estudo e na divulgação do uso do solo e seus efeitos no meio urbano.

\section{REFERÊNCIAS BIBLIOGRÁFICAS}

ACCIOLY, A.M.A.; SIQUEIRA, J.O. Contaminação química e biorremediação do solo. In: NOVAIS, R.F.; ALVARES, V.H.; SCHAEFER, C.E.G.R. Tópicos em ciência do solo, Viçosa : SBCS, 2000. V. 1, p.299-352.

AGASSI, M. Soil erosion, conservation and rehabilitation. New York : Marcel Dekker, 1996. 402p.
ALEXANDROVSKAYA, E.I.; ALEXANDROVSKIY, A.L. History of the cultural layer in Moscow and accumulation of anthropogenic substances in it. Catena, v.41, n.1-3, p.249259,2000 .

ANJOS, M.J. et al. Elemental concentration analysis in soils contaminated with recyclable urban garbage by tube-exited energy-dispersive X-ray fluorescence. Radiation Physics and Chemistry, v.65, p.495-500, 2002.

BAVER, L.D.; GARDNER, W.H.; GARDNER, W.R. Soil physics. New York : John Wiley \& Sons, 1972. 498p.

BERGER, M.G. Uso do sensoriamento remoto na hierarquização das áreas de risco ambiental na subbacia hidrográfica do Arroio Cadena, Município de Santa Maria - RS. Santa Maria, 2001. 144f. Dissertação (Mestrado em Engenharia Agrícola) - Curso de Pós-graduação em Engenharia Agrícola, Universidade Federal de Santa Maria.

BERNARD, A.M. Effects of heavy metals in the environment on human health. In. _____. Contaminated soils, Paris, 1995. Paris : INRA, 1997. p.21-33. (Les colloques, $\mathrm{n}^{\circ} 85$ ).

BLUME, H.P. Classification of soils in urban agglomerations. Catena, v.16, n.3, p.269-275, 1989.

BRASIL. Lei Federal n. 6.939 de 31 de agosto de 1981. Define a política nacional do meio ambiente.

BRADSHAW, A.; HUNT, B.; WALMSLEY, T. Trees in the urban landscape: principles and practices. London : $\mathrm{E}$ \& FN Spon, 1995. 271p.

CRAUL, P.J. Urban soil in landscape design. New York: John Wiley, 1992.

CRAUL, P.J. Urban soils: Applications and practices. New York : John Wiley, 1999. 366p.

D'COSTA, V.P.; OMOTO, W.O.; ONYATTA, J.O. Application of soils and land evaluation data for urban and peri-urban land use planning of Kisumu, Kenya. In. WORLD CONGRESS OF SOIL SCIENCE, 17., 2002, Bangkok, Thailand. Anais... Bangkok, 2002. V.5, p.1670.

DE KIMPE, C.R.; MOREL, J.L. Urban soil management: a growing concern. Soil Science, v.165, n.1, p.31-40, 2000.

DIAS JUNIOR, M.S. Compactação do solo. In: NOVAIS, R.F., ALVARES, V.H.; SCHAEFER, C.E.G.R. Tópicos em ciência do solo. Viçosa : SBCS, 2000. V.1, p.55-94.

EMBRAPA. Centro Nacional de Pesquisa de Solos. Sistema brasileiro de classificação de solos. Brasília : Embrapa, 1999. 412p.

FAO. Soil Map of the World. Revised Legend. Rome: FAO, 1994.

FUNASA - Fundação Nacional de Saúde. Manual de saneamento. 1992. Capturado em 01 de julho de 2003. Online. Disponível na Internet: http://www.funasa.gov.br/pub/ pub00.htm\#

GAIVIZZO, L.H.B. Fracionamento e mobilidade de metais pesados em solo com descarte de lodo industrial. 2001. 123f. Tese (Doutorado em Ciência do Solo) - Programa 
de Pós-graduação em Ciência do Solo, Universidade Federal do Rio Grande do Sul.

GE, Y.; MURRAY, P.; WENDERSHOT, W.H. Trace metal speciation and bioavailability in urban soils. Environmental Pollution, v.107, p.137-144, 2000

GRANDO, M.Z.; MIGUEL, L.A. Agricultura na região metropolitana de Porto Alegre, aspectos históricos e contemporâneos. Porto Alegre : UFRGS, 2000. 157p.

HARRIS, R.W.; CLARK, J.R.; MATHENY, N.P. Arboriculture: integrated management of landscape trees, shrubs, and vines. Upper Saddle River: Prentice Hall, 1999

HILLEL, D. Environmental soil physics. San Diego : Academic, 1998. 771p.

HILLER, D.A. Properties of urbic anthrosols from an abandoned shunting yard in the Ruhr area, Germany. Catena, v.39, n.4, p.245-266, 2000

HUDSON, N. Soil conservation. Ames : ISU, 1995. 391p

ISBELL, R.F. The australian soil classification. Collingwood: CSIRO, 1996. 151p.

ISSSWG RB. International Society of Soil Science Working Group RB. World Reference Base for Soil Resources: Introduction. Leuven: Acco : ISRIC, Rome: FAO, 1998. 79p.

JIM, C.Y. Urban soil characteristcs and limitations for landscape planting in Hong Kong. Landscape and Urban Planning, v.40, p.235-249, 1998

JENNY, H. Factors of soil formation. New York : McGrawHill, 1941. 281p.

LU, Y. et al. Concentrations and chemical speciations of $\mathrm{Cu}$, $\mathrm{Zn}, \mathrm{Pb}$ and $\mathrm{Cr}$ of urban soils in Nanjing, China. Geoderma, v.1976, p.1-11, 2003.

KELLER, E.A. Environmental geology. 7.ed. Upper Saddle River : Prentice Hall, 1996. 560p.

MADRID, L.; DÍAZ-BARRIENTOS, E.; MADRID, F. Distributions of heavy metals contents of urban soils in parks of Seville. Chemosphere, v.49, p.1301-1308, 2002.

MANTA, D.S. et al. Heavy metals in urban soils: a case study from the city of Palermo (Sicily), Italy. The Science of the Total Environmental, v.300, p.229. 243,2002

NAVAS, A.; JAVIER, M. Spatial distribution of heavy metals and arsenic in soils of Aragon (northeast Spain): controlling factors and environmental implications. Applied Geochemistry, v.17, n.8, p.961-973, 2002

OLIVEIRA, A.M.S.; BRITO, S.N.A. Geologia de engenharia. São Paulo : ABGE, 1998.

OLIVEIRA, C.M.G. Carta de risco de colapso de solos para a área urbana do município de Ilha Solteira - PS. 2002. 93f. Dissertação (Mestrado em Engenharia Civil) UNESP.

POPKOV, S.; DEMENT'EVA, E. Soil properties effect on the development and distribution of urban vegetative cover of the forest zone. In. WORLD CONGRESS OF SOIL SCIENCE, 17. 2002, Bangkok, Thailand. Anais... Bangkok, 2002. V.5, p.1672.

REINERT, D.J. Soil structural form and stability induced by tillage in a Typic Hapludalf. 1990. $120 \mathrm{f}$. Tese (Doutorado em Ciência do Solo) - Michigan State University.

ROBAINA, L.E. et al. Análise dos ambientes urbanos de risco do município de Santa Maria - RS. Ciência \& Natura, v.23, p.139-152, 2001

SCHLEUß, U.; WU, Q.; BLUME, H. Variability of soils in urban and periurban areas in Northern Germany. Catena, v.33, p.255-270, 1998 .

SCHUELER, T. The compaction of urban soils. Technical note $\mathrm{n}^{\circ} 107$. Watershed Protection Techniques, v.3, n.2, p.661665, 2000. Capturado em 13 de agosto de 2002. Online. Disponível na Internet http://www.stormwatercenter.net/Practice/36The $\% 20$ Compaction $\% 20$ of $\% 20$ urban $\% 20$ Soils.pdf

SHINN, N.J. et al. Determination of spatial continuity of soil lead levels in an urban residential neighborhood Environmental Research, v.82, p.46-52, 2000.

SIMÃO, J.B.P.; SIQUEIRA, J.O. Solos contaminados por metais pesados: características, implicações e remediações. Recuperação de áreas degradadas, Belo Horizonte, v.22, n.210, p.18-26, mai-jun, 2001. (Informe Agropecuário)

SMYTH, C.G.; ROYLE, S.A. Urban landslide hazard: incidence and causative factors in Niterói, Rio de Janeiro State, Brazil. Applied Geography, v.20, p.95-117, 2000.

SOANE, B.D.; VAN OUWERKERK, C. Soil compaction in crop production. Amsterdam : Elsevier, 1994. 662p.

STROGANOVA, M.N.; AGARKOVA, M.G. Urban soils: experimental study and classification (exemplified by soils of Southwestern Moscow). Eurasian Soil Science, v.25, n.3, p.59-69, 1993.

SPAARGAREN, O.C. Other systems of soil classification. In. SUMMER, M.E. Handbook of soil science. Boca Raton : CRC, 2000. Section E, p.137-174.

USDA-NRCS. Urban soil compaction. Urban Technical Note $N^{\circ}$ 2, march, 2000a. Capturado em 15 de maio de 2002. Online. Disponível na Internet http:/ /www.statlab.iastate.edu/ survey/SQI/pdf/u01d.pdf

USDA-NRCS. Erosion and sedimentation on construction site. Urban Technical Note $\mathrm{N}^{\mathrm{o}} 1$, march, 2000b. Capturado em 15 de maio de 2002. Online. Disponível na Internet http://www.statlab.iastate.edu/ survey/SQI/pdf/u02d.pdf 